\title{
The Relationship between Aerobic Fitness and Change in Repeated Sprint Ability before and after a Half-time
}

\author{
Natsumi Kinoshita $^{1}$, Fuminori Takayama ${ }^{1,2}$, and Yoshiharu Nabekura ${ }^{3}$ \\ ${ }^{1}$ Graduate School of Comprehensive Human Sciences, University of Tsukuba \\ 1-1-1 Tennodai, Tsukuba-shi, Ibaraki 305-8574, Japan \\ ${ }^{2}$ Research Fellow of Japan Society for the Promotion of Science \\ 5-3-1 Kojimachi, Chiyoda-ku, Tokyo 102-0083, Japan \\ ${ }^{3}$ Faculty of Health and Sport Sciences, University of Tsukuba \\ 1-1-1 Tennodai, Tsukuba-shi, Ibaraki 305-8574, Japan \\ E-mail: nabekura.yoshihar.fm@u.tsukuba.ac.jp \\ [Received December 19, 2016; Accepted May 29, 2017; Published online July 7, 2017]
}

\begin{abstract}
This study investigated the relationship between aerobic fitness and repeated sprint ability (RSA) before and after a half-time. Thirteen male volunteers $(23 \pm 2$ years, $175 \pm 5 \mathrm{~cm}, 67 \pm 6$ $\mathrm{kg}$; [mean \pm standard error]) participated in this study. All subjects underwent two laboratory tests on a cycle ergometer. An initial test was conducted to determine aerobic fitness. On separate day, the RSA test was conducted. The RSA exercise comprised $15 \times 6$-s sprints with 54-s recovery periods. This was duplicated after a 15-min half-time. The blood lactate (Bla) removal rate was calculated using Bla values before and after the half-time. As a result of aerobic fitness, seven subjects were classified as fit $(\geq 58 \mathrm{ml} / \mathrm{kg} / \mathrm{min})$ and six as unfit $(<58$ $\mathrm{ml} / \mathrm{kg} / \mathrm{min})$. The maximal peak power was significantly reduced in the unfit group $(\mathrm{p}<0.05)$ but not in the fit group $(p>0.05)$ at the second session. The change in maximal peak power between sessions was significantly correlated with $\dot{V} \mathrm{O}_{2} \max (r=0.714, p<0.01)$. A strong correlation was observed between Bla removal rate and $\dot{V} O_{2} \max (r=0.830, p<0.01)$. These results indicate that aerobically fit subjects recover more quickly after RSA exercise due to superior lactate removal ability.
\end{abstract}

Keywords: blood lactate removal, maximal aerobic power, recovery, team sports

\section{Introduction}

Team sports such as soccer, handball and basketball require athletes to perform a number of sprints, interspersed with intervals of sub-maximal intensity activity (Mohr et al., 2003; Póvoas et al., 2014). The ability to repeat high-intensity activity has been termed repeated sprint ability (RSA). This is important for performance in team sports athletes (Bishop et al., 2011).

Coaches and athletes believe that a high level of aerobic fitness contributes to RSA. However, there is controversy about this relationship. Maximal oxygen uptake ( $\mathrm{V}_{2}$ max) is one of the best indices of an individual's ability to transport oxygen to working muscles (Bassett and Howley, 2000). Several studies have shown that RSA is influenced by $\mathrm{V}_{2}$ $\max$ (McMahon et al., 1998; Jones et al., 2013; da
Silva et al., 2010) whereas some studies reported no significant relationship between $\dot{\mathrm{V}}_{2}$ max and RSA (Angius et al., 2013; Ariz et al., 2007).

It has been reported that RSA performance depends on maximal peak power (Bishop and Spencer, 2004; Hamilton et al., 1991). For example, investigating two groups with similar peak $\mathrm{V}_{2}$ but different maximal peak power, Bishop and Spencer (2004) reported that the percentage decrement (\%DS) for subjects with higher maximal peak power was significantly greater than that of subjects with lower maximal peak power. Thus, it is suggested that differences in subject characteristics may explain the discrepancy in results from previous studies.

Typical team sports games have half-time periods. Many studies using computerised time-motion analysis have demonstrated that the frequency 
of high-intensity running is reduced over the time course of these games (Di Salvo et al., 2009; Mohr et al., 2003; Póvoas et al., 2014), suggesting that RSA performance is decreased after a half-time.

It is well known that onset of fatigue during team sports game coincides with lactate accumulation in working muscles (Krustrup et al., 2006). Lactate accumulation resulted in decline in muscle $\mathrm{pH}$ and inhibition of phosphofructokinase and glycogenolysis (Hollidge-Horvat et al., 1999). Previous studies reported that blood lactate (Bla) level after a single RSA exercise was approximately $10 \mathrm{mmol} / \mathrm{L}$ (Bishop and Spencer, 2004; Gharbi et al., 2014), which indicates that RSA may be reduced during second sessions compared with first sessions. Since oxidative capacity is related to Bla removal ability (Thomas et al., 2004), it is possible that aerobic fitness is associated with the change in RSA before and after a half-time. However, it is unknown whether aerobic fitness affects RSA after a halftime.

This study aimed to reveal the relationship between aerobic fitness and the change in RSA before and after a half-time. We hypothesised that aerobically fit subjects would maintain RSA during a second session of exercise, whereas the RSA of aerobically unfit subjects would be reduced.

\section{Methods}

\subsection{Subjects}

Thirteen male volunteers ( $23 \pm 2$ years, $175 \pm 5$ $\mathrm{cm}, 67 \pm 6 \mathrm{~kg}$; [mean \pm standard error]) participated in this study. All of the subjects were at least moderately active but individual training status (e.g. volume, intensity, frequency) varied. Before participation, all subjects gave informed consent. This study was approved by the Ethical Committee of the University of Tsukuba and was performed in accordance with the Declaration of Helsinki.

Previous study investigated $\mathrm{V}_{2}$ max in 450 elite soccer players to clarify the metabolic compliance of athletes to the filed role requirements, and reported that median value of $\dot{\mathrm{V}}_{2}$ max was $58 \mathrm{ml} / \mathrm{kg}$ / min (Manari et al., 2016). Thus, subjects of the present study were categorised by $\dot{\mathrm{V}}_{2}$ max value as aerobically fit ( $\geq 58 \mathrm{ml} / \mathrm{kg} / \mathrm{min}$ ) or aerobically unfit ( $<58 \mathrm{ml} / \mathrm{kg} / \mathrm{min})$.

\subsection{Experimental design}

Each subject underwent two laboratory tests on a cycle ergometer (Power Max VII, Konami Corporation, Tokyo, Japan). An initial test was conducted to determine aerobic fitness. On a separate day, the RSA test was conducted. Each test was at least 3 days apart (range 3-8 days). Individual tests were performed simultaneously $( \pm 1 \mathrm{~h})$ and in the same experimental room (ambient temperature: 22$24^{\circ} \mathrm{C}$ ). Subjects refrained from heavy training, caffeine and alcohol for $24 \mathrm{~h}$ before each test. During the preceding $4 \mathrm{~h}$, only ad libitum water intake was allowed, and subjects consumed light meals at least $4 \mathrm{~h}$ before the test.

\subsection{Aerobic fitness}

Aerobic fitness was assessed by a sub-maximal graded test and a maximal incremental test. Subjects were instructed to maintain a pedalling rate of $90 \mathrm{rpm}$ with the aid of a metronome. To determine the lactate threshold (LT), the sub-maximal graded test comprised four to six continuous stages of 4 min, with 2 min of rest between stages. A 2-min rest period occurred for blood sampling. The initial load was designed according to each subject's training status and body weight, and thereafter the load was increased progressively by $0.3-0.4 \mathrm{kp}$. The test was terminated when the rating of perceived exertion (RPE) (Borg, 1982) exceeded approximately 15.

Ten minutes after the sub-maximal graded test, the maximal incremental test was performed to determine $\dot{\mathrm{V}}_{2}$ max. The subjects commenced at the load of the penultimate stage of the sub-maximal graded test for 1 minute, and thereafter the load was increased by $0.2 \mathrm{kp}$ each minute until volitional exhaustion (pedalling rate $<85 \mathrm{rpm}$ ). All subjects were verbally encouraged by the same investigators.

Expired gases and ventilation were continuously measured using the computerised standard open circuit technique (AE-310s, Minato Medical Science, Osaka, Japan). The data were averaged into 15 -s intervals. Before each test, oxygen and carbon dioxide analysers were calibrated with known gas concentrations, and flow calibration was performed using a 2-L syringe. Heart rate (HR) was recorded via telemetry (S610s, Polar Oy, Kempele, Finland). Finger tip capillary blood samples were taken at rest and after each stage during the sub-maximal graded 
test and 1, 3 and 5 min after the maximal incremental test. Bla was determined using an automatic lactate analyser (YSI 1500 SPORT, Yellow Springs Instruments, Ohio, USA). Before each measurement, the analyser was calibrated according to the manufacturer's instructions using standard lactate solutions of 0,5 and $15 \mathrm{mmol} / \mathrm{L}$.

LT was determined as the final point before the Bla increased from the resting concentration and was defined as the load where the Bla was 1.0 $\mathrm{mmol} / \mathrm{L}$ above the resting value (Coyle et al., 1983). $\dot{\mathrm{VO}}_{2}$ max was taken as the highest mean of four consecutive 15-s $\dot{\mathrm{V}}_{2}$ values during the maximal incremental test. The $\dot{\mathrm{V}}_{2}$ max was the value that met at least three of the following criteria: (1) a levelling off of $\dot{\mathrm{V}}_{2}$ despite an increase in load, (2) respiratory exchange ratio $\geq 1.05$, (3) HRmax $\geq 95 \%$ of the age predicted HRmax (220-age) and (4) peak Bla concentration $\geq 8 \mathrm{mmol} / \mathrm{L}$. HRmax was taken as the highest 5-s mean during the maximal incremental test. The minimal power that theoretically elicited $\dot{\mathrm{VO}}_{2} \max \left(\mathrm{PVO}_{2} \mathrm{max}\right)$ was calculated as described by previous study (Daniels et al., 1984).

\subsection{Repeated sprint ability test}

The RSA exercise comprised $15 \times 6$-s sprints before and after a 15-min half-time. Before the first session, the subjects performed a 5-min warm-up which comprised 4-min sub-maximal cycling (1 kp $\times 60 \mathrm{rpm}$ ), followed by two maximal sprints for 10 $\mathrm{s}$ at $2 \mathrm{kp}$, separated by a $30-\mathrm{s}$ passive recovery period. Five minutes after the warm-up, the first session was performed. The feet were secured to the pedals with straps. Each subject's starting position, as an individual preference, was controlled by the crank angle throughout the trials.

The RSA exercise comprised $15 \times 6$-s maximal sprints with resistance set at $8 \%$ body weight, separated by 54-s recovery periods (Figure 1). Each

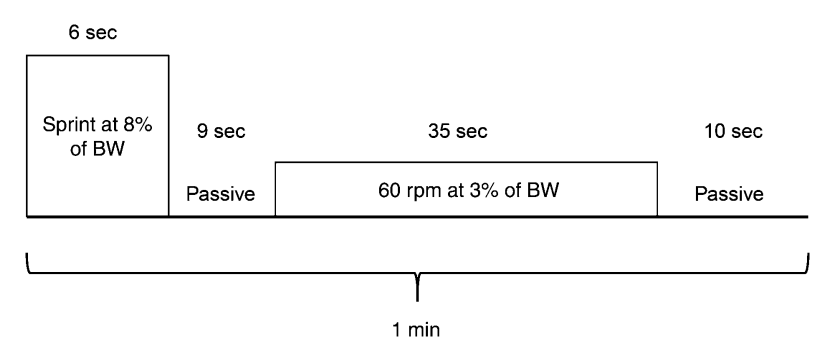

Figure 1 Layout of one set in the repeated sprint ability test. recovery period comprised 35 -s active recovery (3\% body weight, $60 \mathrm{rpm}$ ) and 19-s passive recovery (following orders; 9-s passive, 35 -s active and 10-s passive). The passive periods were in place to stop cycling after the preceding sprint and prepare for the next sprint. The subjects were instructed to avoid conserving energy for the last part of the sprint and stay seated on the ergometer to limit the recruitment of other muscle groups. They were encouraged by same investigators to make a maximal effort during each sprint. Fifteen minutes after the first session, the second session was conducted in the same manner as the first session. The subjects were required to sit in a chair between sessions.

HR was monitored continuously during the test and HRmean was calculated based on HRmax in the aerobic fitness assessment. Bla was measured before the warm-up, after the first session and before and after the second session using the same technique as for the aerobic fitness assessment. The Bla removal rate was calculated using the Bla values after the first session and before the second session, described in the following equation:

Bla removal rate $=($ Bla value after first session - Bla value before second session)/Bla value after first session $\times 100$

The RPE was recorded after each session.

The power data were sampled $(10 \mathrm{~ms})$ and recorded on a personal computer (INSPIRON1300, Dell, Texas, USA) using cycle ergometer software. The following variables were obtained: (a) total work: the sum of the mean power output in each sprint; (b) maximal peak power: the highest value from all sprints; (c) $\% \mathrm{DS}=[100-$ (sum of peak power/maximal peak power $\times 15) \times 100$ ] (Bishop et al., 2004). Maximal peak power was also expressed as the percentage of $\mathrm{PVO}_{2} \mathrm{max}$. These variables were analysed separately for each session.

\subsection{Statistical analysis}

Data are expressed as means with standard errors. Mann-Whitney U tests were used to assess whether significant differences existed between groups. For comparing between sessions, Wilcoxon's signed-rank tests were used. Relationships between $\dot{\mathrm{V}}_{2} \max$ and selected variables were determined using Pearson's product-moment correlation coefficient tests. $\mathrm{p}<0.05$ was considered significant. The statistical analyses were performed with SPSS 
Statistics 22 (IBM Japan, Tokyo, Japan).

\section{Results}

Based on $\dot{\mathrm{V}}_{2} \max$ values, seven subjects were classified as fit and six as unfit. There were no significant differences in height or body weight between the fit and unfit groups $(175.3 \pm 1.8 \mathrm{~cm}$ vs $175.2 \pm 2.2 \mathrm{~cm}, 68.1 \pm 2.4 \mathrm{~kg}$ vs $66.5 \pm 2.2 \mathrm{~kg}$, respectively; $p>0.05$ ).

\subsection{Aerobic fitness}

Aerobic fitness data are shown in Table 1. As expected, absolute and relative $\dot{\mathrm{V}}_{2}$ max values of the fit group were higher than those of the unfit group $(\mathrm{p}<0.01)$. Absolute and relative $\mathrm{PV}_{2} \max$ values were also significantly higher for the fit group $(\mathrm{p}<$ 0.01 ). Although absolute power output at the LT was not significantly different between the groups, the value approached significance $(p=0.051)$. The relative power output at the LT was significantly different $(\mathrm{p}<0.05)$. The $\% \dot{\mathrm{V}}_{2} \mathrm{max}$ at the LT was not significantly different between the groups $(p>$ $0.05)$.

\subsection{Repeated sprint ability test}

Data from the RSA test are shown in Table 2. Although the absolute maximal peak power was not significantly different between the groups, the $\% \mathrm{PVO}_{2}$ max was higher for the unfit group than for the fit group at both sessions $(\mathrm{p}<0.01)$. There were no significant differences in total work between groups at either session $(p>0.05)$. \%DS was sig-

Table 1 Aerobic fitness data.

\begin{tabular}{lcc}
\hline & $\begin{array}{c}\text { Fit group } \\
(\mathrm{n}=7)\end{array}$ & $\begin{array}{c}\text { Unfit group } \\
(\mathrm{n}=6)\end{array}$ \\
\hline$\dot{\mathrm{V}}$ & $4.23 \pm 0.18$ & $3.05 \pm 0.17^{* *}$ \\
$\dot{\mathrm{V}} \mathrm{max}(\mathrm{L} / \mathrm{min})$ & $62.0 \pm 1.0$ & $45.8 \pm 1.8^{* *}$ \\
$\mathrm{PVO}_{2} \mathrm{max}(\mathrm{ml} / \mathrm{kg} / \mathrm{min})$ & $316.4 \pm 15.1$ & $213.0 \pm 10.2^{* *}$ \\
$\mathrm{PV} \dot{\mathrm{O}}_{2} \mathrm{max}(\mathrm{W} / \mathrm{kg})$ & $4.6 \pm 0.1$ & $3.2 \pm 0.1^{* *}$ \\
$\mathrm{LT}(\mathrm{W})$ & $167.2 \pm 18.6$ & $107.5 \pm 12.9$ \\
$\mathrm{LT}(\mathrm{W} / \mathrm{kg})$ & $2.4 \pm 0.2$ & $1.6 \pm 0.2^{*}$ \\
$\mathrm{LT}\left(\% \dot{\mathrm{V}} \mathrm{O}_{2}\right.$ max) & $56.6 \pm 3.0$ & $54.6 \pm 5.4$ \\
$\mathrm{HRmax}($ beats/min) & $194 \pm 4$ & $188 \pm 5$ \\
Peak blood lactate & & \\
$(\mathrm{mmol} / \mathrm{L})$ & $10.1 \pm 0.6$ & $9.4 \pm 0.7$ \\
\hline
\end{tabular}

Data are presented as means \pm standard errors

Significant difference between groups $\left({ }^{*} \mathrm{p}<0.05, * * \mathrm{p}<0.01\right)$ nificantly greater for the unfit group at both sessions compared with that of the fit group.

The HRmean was significantly higher for the unfit group at both sessions than for the fit group ( $p<$ 0.01 ). There were no significant differences in post Bla or post RPE between the groups at either session $(p>0.05)$. The Bla removal rate was significantly higher for the fir group than for the unfit group ( $\mathrm{p}<0.01)$.

When comparing between sessions, there were no changes in maximal peak power or total work for the fit group $(p>0.05)$. However, the maximal peak power of the unfit group was significantly reduced at the second session $(p<0.05)$. Total work was not significantly changed for the unfit group $(p>0.05)$.

\subsection{Relationship between $\dot{\mathbf{V}} \mathbf{O}_{2} \max$ and repeated sprint ability variables}

Maximal peak power (first session: $r=0.214$, second session: $r=0.473$ ) and total work (first session: $r=0.422$, second session: $r=0.548$ ) was not correlated with $\dot{\mathrm{V}} \mathrm{O}_{2} \max (\mathrm{p}>0.05)$. There was a sig-

Table 2 Repeated sprint ability data.

\begin{tabular}{|c|c|c|}
\hline & $\begin{array}{l}\text { Fit group } \\
\quad(n=7)\end{array}$ & $\begin{array}{l}\text { Unfit group } \\
\quad(\mathrm{n}=6)\end{array}$ \\
\hline \multicolumn{3}{|l|}{ Maximal peak power } \\
\hline First (W) & $816 \pm 38$ & $788 \pm 29$ \\
\hline Second (W) & $830 \pm 36$ & $749 \pm 34^{\dagger}$ \\
\hline First $(\mathrm{W} / \mathrm{kg})$ & $12.0 \pm 0.3$ & $11.8 \pm 0.1$ \\
\hline Second $(\mathrm{W} / \mathrm{kg})$ & $12.2 \pm 0.3$ & $11.3 \pm 0.2^{\dagger}$ \\
\hline First $\left(\% \mathrm{PVO}_{2} \max \right)$ & $259 \pm 7$ & $373 \pm 17^{* *}$ \\
\hline Second $\left(\% \mathrm{PVO}_{2} \max \right)$ & $263 \pm 7$ & $354 \pm 15^{* * \dagger}$ \\
\hline \multicolumn{3}{|l|}{ Total work } \\
\hline First (kJ) & $50.6 \pm 4.0$ & $44.2 \pm 2.6$ \\
\hline Second $(\mathrm{kJ})$ & $52.0 \pm 4.0$ & $43.0 \pm 2.2$ \\
\hline \multicolumn{3}{|l|}{ Percentage decrement } \\
\hline First (\%) & $3.6 \pm 0.6$ & $15.3 \pm 2.8^{* *}$ \\
\hline Second $(\%)$ & $5.1 \pm 1.0$ & $11.3 \pm 2.8^{*}$ \\
\hline \multicolumn{3}{|l|}{ Heart rate mean } \\
\hline First (\%HRmax) & $72.4 \pm 2.2$ & $82.0 \pm 2.2^{* *}$ \\
\hline Second (\%HRmax) & $74.7 \pm 1.9$ & $82.2 \pm 1.6^{* *}$ \\
\hline \multicolumn{3}{|l|}{ Blood lactate } \\
\hline After first (mmol/L) & $6.5 \pm 1.1$ & $9.5 \pm 0.8$ \\
\hline Before second $(\mathrm{mmol} / \mathrm{L})$ & $3.8 \pm 0.6$ & $7.5 \pm 0.7$ \\
\hline After second $(\mathrm{mmol} / \mathrm{L})$ & $7.1 \pm 1.2$ & $8.7 \pm 0.8$ \\
\hline Removal rate $(\%)$ & $40.4 \pm 2.5$ & $21.5 \pm 2.2^{* *}$ \\
\hline \multicolumn{3}{|l|}{ Rating of perceived exertion } \\
\hline First & $15.4 \pm 0.8$ & $16.7 \pm 0.4$ \\
\hline Second & $16.3 \pm 0.8$ & $17.8 \pm 0.5$ \\
\hline
\end{tabular}

Data are presented as means \pm standard errors

Significant difference between groups $\left({ }^{*} \mathrm{p}<0.05,{ }^{* *} \mathrm{p}<0.01\right)$

Significant difference between sessions $\left({ }^{\dagger} \mathrm{p}<0.05\right)$ 


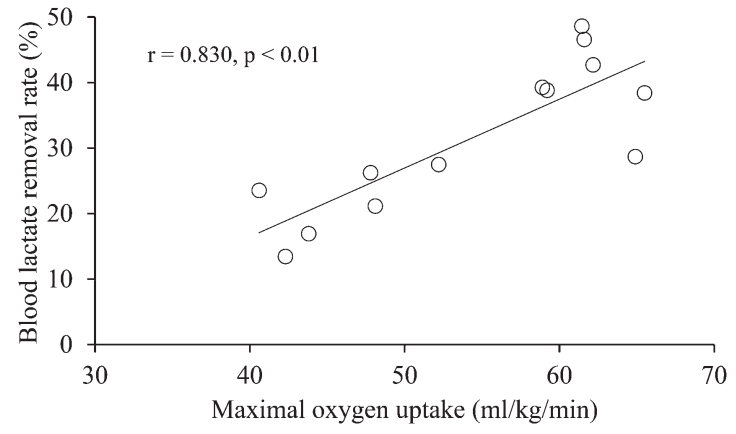

Figure 2 Relationship between maximal oxygen uptake and blood lactate removal rate.

nificant relationship between $\mathrm{V}_{2}$ max and \%DS at the first session $(r=-0.779, p<0.01)$, whereas the relationship at the second session was approaching significance $(r=-0.505, p=0.078)$. At both sessions, HRmean was significantly correlated with $\dot{\mathrm{V}} \mathrm{O}_{2} \max$ (first session: $\mathrm{r}=-0.710$, second session: $\mathrm{r}$ $=-0.757$, respectively; $\mathrm{p}<0.01$ ). Bla removal rate and $\dot{\mathrm{V}}_{2}$ max were strongly correlated $(\mathrm{r}=0.830$, $\mathrm{p}$ $<0.01$; Figure 2). The change in maximal peak power between sessions was significantly correlated with $\dot{\mathrm{V}} \mathrm{O}_{2} \max (\mathrm{r}=0.714, \mathrm{p}<0.01)$, whereas the relationship between change in total work and $\dot{\mathrm{V}}_{2}$ max was not significant $(\mathrm{r}=0.462, \mathrm{p}>0.05)$.

\section{Discussion}

This study aimed to reveal the relationship between aerobic fitness and the change in RSA before and after a half-time. We found that the maximal peak power of the unfit group was significantly reduced at the second session, whereas the performance of the fit group was maintained. In addition, a strong correlation was observed between Bla removal rate and $\dot{\mathrm{V}}_{2} \max$. These results indicate that aerobically fit subjects maintain high performance during second sessions due to superior blood lactate removal ability.

We believe this is the first study to investigate the relationship between aerobic fitness and change in RSA before and after a half-time. The maximal peak power was significantly reduced at the second session in the unfit group, but was maintained in the fit group. In addition, the change in maximal peak power between sessions was significantly correlated with $\dot{\mathrm{V}}_{2}$ max. These results showed that aerobic fitness influenced performance after a half-time. It is well known that accumulated $\mathrm{H}+$ due to a high rate of lactate production contributes to fatigue during maximal effort exercise (Sahlin, 1992). Since oxidative capacity is related to blood lactate removal ability (Thomas et al., 2004), we hypothesised that in aerobically fit subjects, RSA was maintained during the second session, whereas in aerobically unfit subjects it was reduced. The hypothesis is supported by the relationships between $\dot{\mathrm{VO}}_{2}$ max and Bla removal rate and the change in maximal peak power. Taken together, it is suggested that aerobically fit subjects recover more quickly after RSA exercise due to their superior lactate removal ability.

$\mathrm{V}_{2} \max$ and \%DS were significantly correlated. This is in agreement with some previous studies (McMahon et al., 1998; Jones et al., 2013; da Silva et al., 2010), but not others (Angius et al., 2013; Ariz et al., 2007). It has been reported that there is a relationship between maximal peak power and \% DS (Bishop and Spencer, 2004; Hamilton et al., 1991). Thus, equivalent maximal peak power between groups may account for the significant relationship between $\dot{\mathrm{VO}}_{2} \max$ and \%DS. Alternatively, this may be explained by the difference in $\mathrm{PVO}_{2}$ max between the groups. In this study, there was no significant difference in absolute maximal peak power between the groups. Contrary to this, the $\% \mathrm{PVO}_{2}$ max was higher for the unfit group than for the fit group at both sessions. This showed that for aerobically unfit subjects, each sprint caused greater relative physiological stress. Therefore, the combined effect of the maximal peak power and relative intensity during each sprint explains why $\dot{\mathrm{V}}_{2}$ max and \%DS were significantly correlated.

Active recovery load was set by body weight in this study. Consequently, HRmean was significantly higher for the unfit group than for the fit group. In addition, a significant relationship between $\mathrm{V}_{2}$ max and HRmean was observed. These results showed that cardiovascular stress for aerobically unfit subjects was heavily taxed than for fit subjects during recovery period. Yamagishi and Babraj (2016) reported that higher recovery intensity induces impaired peak power restoration during repeated sprints. It is well known that recovery of power output during RSA exercise is correlated with phosphocreatine resynthesis (Bogdanis et al., 1995). Furthermore, Tomlin and Wenger (2001) suggested that good aerobic fitness contributes to recovery from high intensity intermittent exercise due to enhanced phosphocreatine resynthesis. We therefore conclud- 
ed that good aerobic fitness enhances recovery potential after an active recovery period at the same load.

Total work and \%DS were unchanged between sessions for unfit group. As mentioned earlier, it has been reported that maximal peak power is strongly correlated with \%DS (Bishop and Spencer, 2004; Hamilton et al., 1991). This is attributed to greater glycogenolysis rate and subsequent $\mathrm{H}+$ accumulation by higher maximal peak power (Bishop and Edge, 2006). As mentioned earlier, the maximal peak power of the unfit group was significantly reduced, suggesting lower glycogenolysis rate during second sessions. Therefore, no change in total work and \%DS for unfit group at second session can be explained by decrease in maximal peak power.

LT $\left(\% \dot{\mathrm{V}} \mathrm{O}_{2} \max \right)$ was not different between groups. Unlike $\dot{\mathrm{V}} \mathrm{O}_{2} \max$, LT $\left(\% \dot{\mathrm{V}}_{2} \max \right)$ is determined primarily by peripheral factor such as muscle fiber type distribution and fat oxidation capacity. Coyle et al. (1988) reported that LT $\left(\% \dot{\mathrm{VO}}_{2} \max \right)$ was associated with cycling experience years $(r=$ $0.75, \mathrm{p}<0.01$ ) in 14 competitive cyclists (range of cycling experience years: 1-8 years). They speculated that intense cycling training performed for $\sim 5$ years promotes peripheral adaptation. None of the subjects was cyclist in the present study, suggesting lack of peripheral adaptation. Based on the significant difference in $\dot{\mathrm{V}}_{2} \max$ between groups, it seems that peripheral adaptation is less important than central adaptation (i.e., cardiac output) during RSA exercise, at least for the subjects examined present study.

A number of other physiological variables, including muscle glycogen content and buffer capacity, have the potential to influence the RSA. Since aerobically fit subjects have higher capacity of accumulate muscle glycogen (Greiwe et al., 1999) and of tolerate metabolic acidosis (Rampinini et al., 2009), it is likely that these adaptations associated with a high $\dot{\mathrm{V}}_{2}$ max may contribute to high performance during second sessions, rather than a high $\dot{\mathrm{V}}_{2}$ max per se. As muscle biopsies were not obtained in the present study, the above explanation remains a theory.

Important limitations of this study include the RSA test protocol. Considering the HR responses, the protocol was able to simulate physiological stress during sports games (Póvoas et al., 2014;
Scanlan et al., 2012). However, it could not simulate physical stresses such as changes in direction and stop-go movements. Moreover, although significant differences were observed, the small sample size might have affected the results. Further studies are required to investigate RSA before and after a half-time period for larger numbers of subjects with different aerobic statuses, using a sports-specific RSA test (Peterson et al., 2015).

\section{Conclusions}

This study revealed a significant difference in RSA after a half-time between subjects with good aerobic fitness and unfit subjects. When compared with the first session, aerobically fit subjects maintained high performance during the second session, whereas in unfit subjects, performance was reduced. A significant relationship between $\mathrm{V}_{2}$ max and Bla removal rate before and after a half-time was observed. These results indicate that aerobically fit subjects recover more quickly after RSA exercise due to superior lactate removal ability.

\section{References}

Angius, L., Olla, S., Piras, F., Mura, R., Ibba, G., Todde, F., Cominu, M., Pinna, M., Concu, A., and Crisafulli, A. (2013). Indexes of physical capacity and repeated sprint ability of young soccer players. Sport Sci Health, 9: 1-6.

Ariz, A. R., Mukherjee, S., Chia, M. Y., and Teh, K. C. (2007). Relationship between measured maximal oxygen uptake and aerobic endurance performance with running repeated sprint ability in young elite soccer players. J Sports Med Phys Fitness, 47: 401-407.

Bassett, D. R. and Howley, E. T. (2000). Limiting factors for maximum oxygen uptake and determinants of endurance performance. Med Sci Sports Exerc., 32: 70-84.

Bishop, D. and Edge, J. (2006). Determinants of repeatedsprint ability in females matched for single-sprint performance. Eur J Appl Physiol., 97: 373-379.

Bishop, D., Edge, J., and Goodman, C. (2004). Muscle buffer capacity and aerobic fitness are associated with repeatedsprint ability in women. Eur J Appl Physiol., 92: 540-547.

Bishop, D., Girard, O., and Mendez-Villanueva, A. (2011). Repeated-sprint ability-part II: recommendations for training. Sports Med., 41: 741-756.

Bishop, D. and Spencer, M. (2004). Determinants of repeatedsprint ability in well-trained team-sport athletes and endurance-trained athletes. J Sports Med Phys Fitness, 44: 1-7.

Bogdanis, G. C., Nevill, M. E., Boobis, L. H., Lakomy, H. K., and Nevill, A. M. (1995). Recovery of power output and muscle metabolites following $30 \mathrm{~s}$ of maximal sprint cycling in man. J Physiol., 482: 467-480.

Borg, G. A. (1982). Psychophysical bases of perceived exertion. Med Sci Sports Exerc., 14: 377-381.

Coyle, E. F., Coggan, A. R., Hopper, M. K., and Walters, T. 
J. (1988). Determinants of endurance in well-trained cyclists. J Appl Physiol., 64: 2622-2630.

Coyle, E. F., Martin, W. H., Ehsani, A. A., Hagberg, J. M., Bloomfield, S. A., Sinacore, D. R., and Holloszy, J. O. (1983). Blood lactate threshold in some well-trained ischemic heart disease patients. J Appl Physiol., 54: 18-23.

Daniels, J., Scardina, N., Hayes, J., and Foley, P. (1984). Elite and subelite female middle-and long-distance runners. Sport Elite performers, 3: 57-72.

da Silva, J., Guglielmo, L. G., and Bishop, D. (2010). Relationship between different measures of aerobic fitness and repeated-sprint ability in elite soccer players. J Strength Cond Res., 24: 2115-2121.

Di Salvo, V., Gregson, W., Atkinson, G., Tordoff, P., and Drust, B. (2009). Analysis of high intensity activity in Premier League soccer. Int J Sports Med., 30: 205-212.

Gharbi, Z., Dardouri, W., Haj-Sassi, R., Castagna, C., Chamari, K., and Souissi, N. (2014). Effect of the number of sprint repetitions on the variation of blood lactate concentration in repeated sprint sessions. Biol Sport, 31: 151-156.

Greiwe, J. S., Hickner, R. C., Hansen, P. A., Racette, S. B., Chen, M. M., and Holloszy, J. O. (1999). Effects of endurance exercise training on muscle glycogen accumulation in humans. J Appl Physiol., 87: 222-226.

Hamilton, A. L., Nevill, M. E., Brooks, S., and Williams, C. (1991). Physiological responses to maximal intermittent exercise: differences between endurance-trained runners and games players. J Sports Sci., 9: 371-382.

Hollidge-Horvat, M., Parolin, M. L., Wong, D., Kones, N. L., and Heigenhauser, G. J. (1999). Effect of induced metabolic acidosis on human skeletal muscle metabolism during exercise. Am J Physiol., 277: E647-E658.

Jones, R. M., Cook, C. C., Kilduff, L. P., Milanović, Z., James, N., Sporiš, G., Fiorentini, B., Fiorentini, F., Turner, A., and Vučković, G. (2013). Relationship between repeated sprint ability and aerobic capacity in professional soccer players. ScientificWorldJournal, 2013: 952350.

Krustrup, P., Mohr, M., Steensberg, A., Bencke, J., Kjaer, M., and Bangsbo, J. (2006). Muscle and blood metabolites during a soccer game: implications for sprint performance. Med Sci Sports Exerc., 38: 1165-1174.

Manari, D., Manara, M., Zurini, A., Tortorella, G., Vaccarezza, M., Prandelli, N., Ancelotti, D., Vitale, M., Mirandola, P., and Galli, D. (2016). $\mathrm{VO}_{2} \mathrm{Max}$ and $\mathrm{VO}_{2} \mathrm{AT}$ : athletic performance and field role of elite soccer players. Sport Sci Health, 12: 221-226.

McMahon, S. and Wenger, H. A. (1998). The relationship between aerobic fitness and both power output and subsequent recovery during maximal intermittent exercise. J Sci Med Sport, 1: 219-227.

Mohr, M., Krustrup, P., and Bangsbo, J. (2003). Match performance of high-standard soccer players with special reference to development of fatigue. J Sports Sci., 21: 519-528.

Peterson, B. J., Fitzgerald, J. S., Dietz, C. C., Ziegler, K. S., Ingraham, S. J., Baker, S. E., and Snyder, E. M. (2015). Aerobic capacity is associated with improved repeated shift performance in hockey. J Strength Cond Res., 29:
$1465-1472$

Póvoas, S. C., Ascensão, A. A., Magalhães, J., Magalhães, J., Seabra, A. F., Krustrup, P., Soares, J. M., and Rebelo, A. N. (2014). Analysis of fatigue development during elite male handball matches. J Strength Cond Res., 28: 2640-2648.

Rampinini, E., Sassi, A., Morelli, A., Mazzoni, S., Fanchini, M., and Coutts, A. J. (2009). Repeated-sprint ability in professional and amateur soccer players. Appl Physiol Nutr Metab., 34: 1048-1054.

Sahlin, K. (1992). Metabolic factors in fatigue. Sports Med., 13: 99-107.

Scanlan, A. T., Dascombe, B. J., Reaburn, P., and Dalbo, V. J. (2012). The physiological and activity demands experienced by Australian female basketball players during competition. J Sci Med Sport, 15: 341-347.

Thomas, C., Sirvent, P., Perrey, S., Raynaud, E., and Mercier, J. (2004). Relationships between maximal muscle oxidative capacity and blood lactate removal after supramaximal exercise and fatigue indexes in humans. J Appl Physiol., 97: 2132-2138.

Tomlin, D. L. and Wenger, H. A. (2001). The relationship between aerobic fitness and recovery from high intensity intermittent exercise. Sports Med., 31: 1-11.

Yamagishi, T. and Babraj, J. (2016). Influence of recovery intensity on oxygen demand and repeated sprint performance. J Sports Med Phys Fitness, 56: 1103-1112.

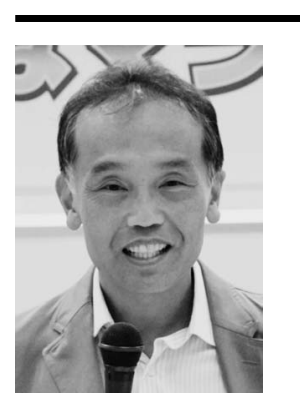

Name:

Yoshiharu Nabekura

Affiliation:
Faculty of Health and Sport Sciences,
University of Tsukuba

Address:

1-1-1 Tennodai, Tsukuba-shi, Ibaraki 305-8574, Japan

Brief Biographical History:

Yoshiharu Nabekura is the professor of Faculty of Health and Sport Sciences at University of Tsukuba.

He obtained Ph.D. at University of Tsukuba in 1991.

Main works:

-Tanji, F., Shirai, Y., Tsuji, T., Shimazu, W., and Nabekura, Y. (2017). Relation between 1,500-m running performance and running economy during high-intensity running in welltrained distance runners. J Phys Fitness Sports Med, 6: 41-48.

Membership in learned Societies:

- Society for Running

- Japan Society of Exercise and Sports Physiology

- Japan Society of Physical Education, Health and Sport Sciences

- Japanese Society of Physical Fitness and Sports Medicine 